\title{
Psychophysiological and Cultural Correlates Undermining a Survivalist Interpretation of Near-Death Experiences
}

\author{
Keith Augustine, M.A. \\ Internet Infidels, Colorado Springs, $\mathrm{CO}$
}

\begin{abstract}
This paper, Part III of a critique of survivalist interpretations of near-death experiences (NDEs), considers psychophysiological and cultural correlates of NDEs suggesting that such experiences are solely products of individuals' minds rather than windows into a transcendental realm. While current psychophysiological models do not fully explain out-of-body experiences (OBEs) and NDEs, several psychophysiological correlates offer promising clues about the mechanisms implicated in their production. These correlates do not definitively identify their precise causes, but strongly imply that such experiences represent internally generated fantasies rather than genuine perceptions of a transcendental environment. Additionally, stark differences in the phenomenology of NDEs from different cultures have been uncovered. Though nonWestern samples are too limited to draw anything more than tentative conclusions, the available data suggest that prototypical Western NDE motifs derive from a cultural source, consistent with the hypothesis that NDE content reflects social conditioning and personal expectation rather than the perception of an external reality.
\end{abstract}

KEY WORDS: near-death experiences; dissociation; temporal lobe signs; cultural variation; expectation.

Keith Augustine, M.A., is Executive Director and Scholarly Paper Editor of Internet Infidels, a nonprofit educational organization dedicated to defending and promoting metaphysical naturalism on the Internet. He would like to thank Oliver Carter, lecturer of English as a Foreign Language at Matsumoto University, for his research assistance and translation of Rinshi Taiken [Near Death Experience] by Takashi Tachibana. Reprint requests should be addressed to Mr. Augustine at Internet Infidels, PO Box 142, Colorado Springs, CO 80901-0142; e-mail: kaugust@infidels.org. 


\section{Psychophysiological Correlates}

Few dispute that out-of-body experiences (OBEs) and near-death experiences (NDEs) are altered states of consciousness (ASCs), temporary departures from the normal (alert) waking state. During ASCs, a variety of mental faculties appear to be altered, including arousal, attention, perceptual functions, imagery skills, memory, cognition, and sense of identity. Sometimes ASCs follow a period of unconsciousness, but they are often triggered during normal consciousness.

ASCs are typically understood to involve turning one's attention inward, into the contents of one's own mind, rather than revealing anything about the external world. For example, dreams are ASCs that are typically understood to call up internally generated imagery; that is, the world encountered during dreaming is not typically taken to involve any real terrain. Similarly, to say that alien abduction experiences involve ASCs (for example, sleep paralysis) is to imply that such experiences do not really involve extraterrestrials physically kidnapping unlucky human subjects. Thus, insofar as OBEs and NDEs are such altered states, there is a reasonable presumption that they do not reflect any objective existence outside of the normal physical body. But specific psychological traits of OBErs and NDErs also strongly imply that some psychophysiological model of OBEs and NDEs must be correct.

For instance, statistically significant correlations between dissociation and absorption and both OBEs and NDEs have been discovered. Dissociation is the shutting out of sensory stimuli, while absorption is focusing on the imagination - but they tend to go hand-in-hand (Greyson, 2000). The classic example of dissociation is "highway hypnosis," daydreaming or consciously focusing on anything other than driving and yet still arriving at your destination, evidently subconsciously engaging in the required driving skills. Nonpathological dissociation exists on a continuum best measured by absorption, the capacity to become highly engrossed in the imagination, including through books or movies (Blackmore, 1993a; Irwin, 2000).

Across studies, dissociation has been consistently positively correlated with OBEs, and a moderate correlation between absorption and OBEs has been uncovered (Alvarado, 2000). OBErs most frequently dissociate from bodily sensations, and those who experience multiple OBEs are particularly prone to such somatoform dissociation (Irwin, 
2000). Harvey Irwin (1993) found that NDErs were more likely to have suffered childhood trauma than nonNDErs, and consequently theorized that NDErs are predisposed to dissociate during unexpected highly stressful situations in order to "escape" from the pain or anxiety of their environments. Similarly, Kenneth Ring and Christopher Rosing (1990) found that NDErs have significantly greater dissociative tendencies than nonNDErs, and suggested that childhood trauma makes victims more prone to dissociation and thus NDEs.

In multiple studies, OBErs have been found to have a higher capacity for absorption (Irwin, 1985a; Glicksohn, 1990; Myers, Austrin, Grisso, and Nickeson, 1983) and a greater need for absorbing experiences (Irwin, 1985a; Irwin, 1985b) than nonOBErs. Moreover, Irwin discovered that highly absorbed people can artificially induce OBEs more easily than people who are not particularly absorbed (Irwin, 2000). Similarly, NDErs have not only been found to have a higher capacity for absorption than nonNDErs (Twemlow and Gabbard, 1984), but to have higher levels of absorption the "deeper" their NDEs (Council, Greyson, and Huff, 1986). Interestingly, Stuart Twemlow and Glen Gabbard (1984) also found that NDErs were more absorbed than those who had OBEs in non-life-threatening conditions. This is not surprising given that NDEs are typically more elaborate than OBEs in other contexts.

Other studies have found that fantasy-proneness is higher among OBErs than nonOBErs (Alvarado and Zingrone, 1994; Hunt, Gervais, Shearing-Johns, and Travis, 1992; Myers, Austrin, Grisso, and Nickeson, 1983; Wilson and Barber, 1983), and that NDErs were not only more fantasy-prone than nonNDErs (Twemlow and Gabbard, 1984), but more highly fantasy-prone the deeper their NDEs (Council, Greyson, and Huff, 1986). Fantasy-proneness is "characterized by a strong investment in fantasy life, vivid hallucinatory ability, intense sensory experience, and excellent eidetic [vivid visual] memory" (Greyson, 2000, p. 324).

Finally, compared to nonOBErs, OBErs tend to have more hallucinatory experiences (McCreery and Claridge, 1995), perceptual distortions (McCreery and Claridge, 1996), distortions of body image (Murray and Fox, 2005), illusory experiences of changes in body size, and floating sensations (Blackmore, 1984). Overall, the best predictors of OBEs are dissociation, hypnotic susceptibility, absorption, and fantasy proneness (Alvarado, 2000).

Although Ring and Rosing (1990) viewed dissociative tendencies as a psychological defense mechanism to "tune out" physical threats to 
one's well-being while simultaneously opening a door to "alternate realities," their hypothetical status as a defense mechanism makes much more sense if OBEs and NDEs do not literally involve any form of disembodiment. For instance, both OBErs and NDErs tend to have high rates of absorption, a trait that Irwin noted "might usefully be thought of as a capacity for imaginative involvement" (Irwin, 2000, p. 263). But whether it is indicative of a predisposition to have OBEs and NDEs or simply a consequence of having them, such a correlation makes little sense if something actually leaves the body during such experiences. For why would psychological mechanisms - as opposed to physiological crises alone - trigger the release of the soul? Conversely, why would literal separation from the body make individuals more prone to fantasize?

Similarly, why should fear alone trigger NDEs, or the sudden onset of fear during OBEs terminate such experiences (as in Irwin, 1985a, p. 81), unless their origins are entirely psychophysiological? As Irwin noted:

The sight of the physical body from the outside may terminate the experience, ... because of the emotional response (fear, contempt, revulsion) this sight evokes, but also perhaps because attention is diverted back to the body. Another factor commonly bringing an end to the OBE is the physical body's being touched by someone. (Irwin, 1999, p. 222)

Irwin added that the fact that the vast majority of OBEs were preceded by physical inactivity implied "that a lack of somatic (kinesthetic and proprioceptive) stimulation is an important condition for the OBE" (1999, p. 227). A psychophysiological process makes sense of why some OBEs originate and end due to emotional reactions or the attention given to bodily processes. But if something literally detaches from the body during OBEs, on the face of it psychological factors should neither trigger its release nor hasten its return.

More troubling still for the notion that something leaves the body are various correlations between imagery skills and OBEs. Compared to nonOBErs, for instance, Susan Blackmore found that OBErs "are better at detecting the viewpoint from which a three-dimensional object is seen and are better able to switch viewpoints in their imagination" (1993a, p. 180), while Anne Cook and Irwin (1983) found that OBErs more accurately anticipated the appearance of physical objects when viewed from different perspectives. In addition, Blackmore's OBErs not only reported "clearer and more detailed" imagined viewpoints than her nonOBErs, but were most significantly better than her nonOBErs at "the ability to imagine the room from a position 
on the ceiling above their own head" (1987, p. 61). Moreover, those who habitually dream in a bird's-eye view or see themselves during their dreams are more likely to have OBEs (Blackmore, 1987; Irwin, 1986). Furthermore, those who can induce OBEs at will have better dream control skills than spontaneous OBErs, a fact anticipated by psychophysiological models given that OBE adepts would be expected to have "mastered" the required psychological abilities compared to "novice" spontaneous OBErs (Alvarado, 2000). Similarly, Irwin (1985a) found that OBErs who reported more control over their OBEs tended to have better somatic imagery skills than more "passive" OBErs. Blackmore nicely summarized most of the findings:

OBErs are better than others at switching from one viewpoint to another (especially to the viewpoint above the head), [are] more proficient at producing clear and detailed images from different viewpoints, and tend to use the observer viewpoint in dream recall.... [T] he easiest switch is to a viewpoint above the head, and ... an observer viewpoint is more often used in recalling unpleasant dreams. (Blackmore, 1987, p. 64)

Blackmore interpreted the greater prevalence of a bird's-eye view in OBErs' unpleasant dreams as "support for the idea that perspective is used as an escape from unpleasant situations" (1987, p. 64) among those better at switching perspective, particularly during "cases of severe pain, shock or fear [where] there is a strong incentive to dissociate oneself from the source of the pain" (p. 57). Additional support comes from Twemlow and Gabbard's (1984) finding that among NDErs, those in severe pain immediately prior to their NDEs felt more detached from their bodies, were more likely to "see" their bodies from afar, and were more likely to "occupy" the environment surrounding their bodies.

That various medical factors affect the content of NDEs is equally difficult to reconcile with the notion that NDEs are transcendental journeys. Studies of medical influences on NDE content have found that cardiac arrest NDEs are more likely to feature a sense of power and an awareness of deceased persons, while NDEs resulting from accidents are more likely to feature feelings of joy and little desire to return to the body (Twemlow and Gabbard, 1984; Twemlow, Gabbard, and Coyne, 1982). A greater sense of dying among cardiac arrest NDErs might trigger visions of the deceased, while unexpected accidents trigger maximally "escapist" detached indifference.

Plausible psychophysiological reasons for other correlations are not as easy to conjecture, but no less problematic for survivalist 
interpretations. For example, NDEs during cardiac arrest or while anesthetized are more likely to feature tunnel experiences and experiences of light (Drab, 1981; Owens, Cook, and Stevenson, 1990; Twemlow and Gabbard, 1984; Twemlow, Gabbard, and Coyne, 1982), while NDEs resulting from the perception of imminent threats (for example, in mountain-climbing accidents) in the absence of actual medical crises more often feature feelings of euphoria, thought speeding up, time slowing down, and life review (Noyes and Kletti, 1976; Stevenson and Cook, 1995). In one study, NDErs under the influence of drugs were also more likely than other NDErs to report an awareness of and communication with other beings, suggesting that drugs directly altered the content of their experiences (Twemlow and Gabbard, 1984). But if NDEs occur when consciousness is released from the confines of the brain, then altering brain chemistry ought not have an effect on NDE content.

Of course, none of these findings are inconsistent with the view that something leaves the body during OBEs and NDEs. But they are clearly not predicted by that view, whereas such correlations are anticipated by psychophysiological models. Those who insist upon a survivalist interpretation of such experiences can surely fit these findings within their particular theoretical frameworks. But on the face of it, existing studies of the traits of OBErs and NDErs make psychophysiological models of such experiences much more likely to be true than their transcendental alternatives.

Whichever model turns out to be correct, a preponderance of the evidence suggests that it will be a psychophysiological one. For if OBEs and NDEs truly represent the detachment of a person's soul from his body, why would people with certain psychological characteristics have a greater ability to leave the body? If some mechanism can be activated to achieve such a detachment, why would that mechanism have any connection to purely psychological traits? The "projection theory" does not make much sense of the extensive psychological data collected on OBErs and NDErs. But those data clearly make sense on a psychophysiological understanding of such experiences.

\section{Is the Temporal Lobe Implicated in NDEs?}

There have long been indications that the temporal lobe is implicated in NDEs. That OBEs could be induced by electrical stimulation of the temporal lobe has been known at least since 1941 
(Tong, 2003). However, the phenomenology of electrically stimulated "OBEs of neurological origin" is clearly distinct from that of spontaneous OBEs. Unlike most spontaneous OBEs, electrical stimulation of the brain tends to produce OBEs in which patients perceive only parts of their bodies from above and perceive clearly illusory distortions and movements of those parts, where patients do not see the environment surrounding their bodies, and in which the experience lacks the realism, continuity, and stability characteristic of spontaneous OBEs (Holden, Long, and MacLurg, 2006; Neppe, 2002). Moreover, spontaneous OBEs seem just as likely to occur in unconscious subjects as conscious ones, but if electrically stimulated OBEs depend upon a conflict between information from the vestibular and visual senses, electrically stimulated OBErs would evidently need to be conscious and perceiving their surroundings with open eyes. And Frank Tong observed that for a patient of Olaf Blanke "stimulation applied while the patient's eyes were closed elicited reports of shifts in perceived body position but failed to elicit out-of-body experiences" (2003, p. 105).

Vernon Neppe (2002) noted that he had previously established the existence of four distinct kinds of déjà vu - the common spontaneous form of associative déjà vu, subjective paranormal déjà vu experiences, temporal lobe epilepsy déjà vu, and déjà vu accompanying schizophrenia - and hypothesized that there may be phenomenologically distinct subtypes of OBEs as well. Perhaps spontaneous OBEs, OBEs induced by relaxation techniques, near-death OBEs, and electrically stimulated OBEs are variants generated by different physiological mechanisms. Alternatively, distinct subtypes might reflect the activation of different parts of a single complex neural mechanism for generating OBEs. On the former hypothesis, multisensory disintegration would be irrelevant to spontaneous, induced, and near-death OBEs, but on the latter, it may well play a role in their production.

While electrically stimulated OBEs may be phenomenologically distinct from spontaneous ones, realistic, continuous, stable, ecstatic, and otherwise apparently indistinguishable OBEs have been reported accompanying temporal lobe seizures (Devinsky, Feldmann, Burrowes, and Bromfield, 1989; Vuilleumier, Despland, Assal, and Regli, 1997). Like spontaneous cases, OBEs evoked by temporal lobe seizures often incorporate accurate reconstructions of events surrounding OBErs' bodies (for example, case 7 , and patients 33,39 , and 40 , in 
Devinsky, Feldmann, Burrowes, and Bromfield, 1989, p. 1082, 1086, respectively; and in a single epileptic who had recurring OBEs for over 10 years in Vuilleumier, Despland, Assal, and Regli, 1997, p. 116), as well as occasional small but significant out-of-body discrepancies (for example, case 4 in Devinsky, Feldmann, Burrowes, and Bromfield, 1989 , p. 1081, where an epileptic OBEr would see his body in the correct clothing but with combed hair even when he knew it to be uncombed beforehand).

Additionally, Christopher French noted that peace or euphoria and mystical feelings "are often reported by temporal lobe epileptics just prior to a seizure," quoting a patient stating that nonepileptics "can't imagine the happiness that we epileptics feel during the second before our attack" (French, 2005, p. 358). Some epileptics "even see apparitions of dead friends and relatives" (Blackmore, 1993a, p. 206). So several NDE elements - including feelings of peace, OBEs, a sense of timelessness, life reviews, and encounters with others suggest a role for the temporal lobe in the phenomenology of the experience (French, 2005).

Bruce Greyson has argued that "key features of NDEs ... have not in fact been reported either in clinical seizures or in electrical stimulation of [temporal lobe] brain structures" (Greyson, 2000, p. 335). But the observations above illustrate that many key features of NDEs are found in such circumstances. Moreover, no one is claiming that NDEs "just are" temporal lobe seizures, but rather that similar temporal lobe activity, in conjunction with other activity in a dying brain, is implicated in NDEs (for example, Blackmore, 1993a, pp. 215-216).

Mark Fox noted that complete NDE accounts from the Religious Experience Research Centre (RERC) archives revealed signs of hypergraphia, a compulsion to write extensively about spiritual realities. One man reported an $\mathrm{OBE}$, a tunnel experience, encounters with deceased relatives, and a life review, followed by 11 pages of speculative hypergraphic testimony about the meaning of life, the purpose of existence, the soul, and the beginning of the universe (Fox, 2003). Fox concluded that:

hypergraphia has long been recognized as a symptom of temporal lobe epilepsy. Whilst the presence of hypergraphia within NDErs' reports has been hidden from view often because of the editing of accounts carried out by near-death researchers themselves, an examination of original accounts ... reveals that hypergraphia is a significant feature of a significant number of accounts. (Fox, 2003, p. 161) 
Plausibly, OBErs and NDErs have greater dissociative tendencies than control groups because they are predisposed to dissociate. Dissociative tendencies, in turn, are correlated with nonpathological temporal lobe instability as measured by various temporal lobe signs (Richards and Persinger, 1991). This suggests that those with greater temporal lobe instability may be more prone to have NDEs than others, a hypothesis that is testable in any number of ways, one of which was suggested by Blackmore, who predicted "that the highest level of temporal lobe signs would be found in those who have NDEs when not medically near death, next highest in those who have NDEs near death and lowest in those who come close to death but have no NDE" (1993a, p. 218). Though circumstantial, evidence of temporal lobe instability among NDErs comes from findings that NDErs report undergoing more mystical experiences than average prior to their NDEs (Greyson and Stevenson, 1980), and having more OBEs and "psychic" experiences than nonNDErs (Kohr, 1983; Makarec and Persinger, 1985). Greyson and Ian Stevenson (1980) also found that perceptions of time distortion were significantly correlated with ecstatic feelings during NDEs. These elements are associated with temporal lobe instability in nonNDE contexts, and there is no other apparent reason for them to be correlated in NDEs.

Recently, Willoughby Britton and Richard Bootzin carried out a more systematic investigation. Their NDE group included subjects who had experienced "life-threatening physical distress as the result of an accident or other injury and [received] a minimum score of 7 on the Near-Death Experience Scale" (Britton and Bootzin, 2004, p. 254). Their nonNDE control group consisted of age- and gender-matched individuals who had not come close to death and who had scored below 7 on Greyson's NDE Scale. Despite having used a less-than-ideal control group, their findings confirmed a role for the temporal lobe in the production of NDEs.

First, NDErs were about four times as likely to have temporal lobe epileptiform electroencephalographic (EEG) activity during sleep than nonNDErs.

Second, NDErs "reported significantly more temporal lobe symptoms" than nonNDErs, such as "frequent anomalous experiences, sleepwalking, olfactory hypersensitivity, and hypergraphia," and scored significantly higher than nonNDErs on a measure of epileptic signs typical of temporal lobe activity, including "feelings of intense 
personal significance and unusual olfactory, auditory, or visual perceptual experiences" (Britton and Bootzin, 2004, p. 255).

Third, not only did NDErs take longer to enter rapid eye movement (REM) sleep than nonNDErs, but there was a positive correlation between the depth of a subject's NDE (as measured by the NDE scale) and the delay in REM sleep onset, so that the deeper the NDE, the greater the delay in the onset of REM sleep.

Fourth, NDErs scored significantly higher on measures of coping skills than nonNDErs.

Fifth, NDErs scored significantly higher than nonNDErs on the Amnestic subscale of the Dissociative Experiences Scale, which measures things like not remembering how you arrived at your destination. Thus NDErs show a greater (nonpathological) tendency to dissociate than nonNDErs.

Britton and Bootzin's findings would have been more decisive if they had used the ideal control group, nonNDErs who came close to death. It is possible that the general trauma of coming close to death - not whether subjects had an NDE - is what accounted for the differences between the two groups. However, they provided substantial evidence that it was the occurrence of the NDE itself that was correlated with these differences. This implied that certain people were physiologically prone to have NDEs.

First, there was a significant correlation between NDE Scale scores and both the temporal lobe epileptiform EEG activity and the delayed onset of REM sleep; but these characteristics were not correlated with any of the collected scores for measures of trauma. If coming close to death had caused the epileptiform EEGs in the NDE group, then those EEGs should have been correlated "with trauma-related factors to a similar degree," but they were not (Britton and Bootzin, 2004, p. 256). Instead, left temporal lobe activity was more highly correlated with NDE Scale scores than with scores on measurements of posttraumatic stress disorder or dissociative disorders, or with a history of head trauma.

Second, a regression analysis found that head trauma was unrelated to epileptiform EEG activity. Additionally, the general incidence of epileptiform EEG activity in those who become unconscious without head trauma is 5.7 percent, as compared to the significantly higher rate of 22 percent in Britton and Bootzin's NDE group. Third, that the epileptiform EEG activity in the NDE group was almost entirely limited to the left temporal lobe was atypical of any other group, 
implying that NDErs were neurophysiologically unique. Finally, unlike typical trauma survivors, the physiological differences between the NDE group and the nonNDE control group "were not associated with negative stress reactions" but "instead with positive coping styles" (Britton and Bootzin, 2004, p. 258). A preponderance of the evidence, then, implicated the temporal lobe in the production of NDEs.

\section{Cultural Differences}

In the industrialized West, where Christian imagery dominates, people who encounter religious figures in their NDEs typically encounter Christian religious figures who appear as they are standardly depicted. For example, one woman who reported seeing a light form above her bedroom door just before "floating" over her body said: "Jesus came walking up to me with arms outstretched. He was dressed in a long white robe, his hair to his shoulders, ginger-auburn, and he had a short beard" (Fenwick and Fenwick, 1997, p. 62). By contrast, the dominant religious imagery of India is Hindu, and predictably NDEs from India typically involve encounters with recognizably Hindu religious figures, such as Yamraj (the Hindu god of death) or his black messengers. One Indian NDEr, for example, reported that "Yamraj was there sitting on a high chair with a white beard and wearing yellow clothes" (Pasricha and Stevenson, 1986, p. 167).

Moreover, while Western NDErs tend to encounter dead friends and relatives more often than religious figures, Hindu religious figures nearly always appear in NDEs from India. Finally, while Western NDErs are often "sent back" in order to take care of immediate family or for some assumed but unknown purpose, NDErs from India report meeting clerks in an impersonal afterlife bureaucracy who send them back because they have been sent the wrong person due to paperwork mistakes (Pasricha and Stevenson, 1986).

Cultural conditioning is evident even in children's NDEs. In the West, children's encounters with other beings during NDEs tend to be more imaginative than those of adults. Morse paraphrased one girl's encounter with Jesus:

He was in a very bright light that she could not describe other than by saying that it made her feel good. He was sitting there with a round belly and a red hat, looking like Santa Claus. (Morse and Perry, 1992, p. 125) 
In other cases children have reported encountering wizards, doctors, guardian angels, living teachers, living playmates, pets, and other animals during NDEs (Morse, 1994).

Todd Murphy translated into English 11 published accounts of NDEs in Thailand, three of which reported two NDEs from the same subject, yielding a total of 14 cases (www.altered-states.net). Because one of the published accounts was so similar to another, Murphy analyzed 10 of them in his paper in this Journal (Murphy, 2001; T. Murphy, personal communication, January 24, 2006). Like NDEs from India, Thai NDEs contrasted starkly with those reported in the West.

For instance, like Indian NDErs, Thai NDErs were far more likely to encounter religious figures than deceased friends and relatives: in 9 out of 10 accounts Thai NDErs met Yamatoots, messengers of the god of death Yama. And in half of the accounts Thai NDErs reported "being told that they were the wrong person, and being ordered back to life" (Murphy, 2001, p. 175).

While OBEs are rather common early on in Western NDEs, being visited by a Yamatoot is "the most common initial phase" of Thai NDEs (Murphy, 2001, p. 170). Where OBEs did occur, "OBEs in Thai NDEs tend immediately to precede meetings with Yamatoots" (2001, p. 171). Tunnels were "largely absent in Thai NDEs," and feelings of peace or euphoria and experiences of light have not been reported (2001, p. 172). Thai NDErs were sometimes judged, but their deeds were recounted by reviewing written records of their lives or the testimony of others. The following case is typical:

I ... found myself in the judgment hall of Yama's palace. I knew that they were ready to judge me for my sins. A giant rooster appeared who told Yama that I had killed him. He emphasized that I had tried to kill him again and again. The rooster also said that he remembered me exactly. An entire flock of roosters also [appeared] and testified that I had killed them, as well. I remembered my actions, and I had to admit that the roosters had told the truth. Yama said that I had committed many sins, and sentenced me to many rebirths both as a chicken, and many other types of birds as well... But, quite suddenly, an enormous turtle appeared. It screamed at Yama, saying "Don't take him; he is a good human, and should be allowed to live." Yama answered the turtle "What did he do to help you?" (Murphy, 2001, p. 167; ellipses in the original)

Unlike Western cases, life events were not viewed or relived as flashbacks. Landscapes were common in these Thai NDEs, but typically hellish. And while "Western NDErs may reach a 'point of 
no return' and choose to return to life, Thai NDErs are typically told they were taken because of a clerical mistake and told to return to the body" (Murphy, 2001, p. 177).

Given such stark phenomenological differences, Murphy concluded: "The fact that Thai (and Indian) NDEs do not follow the typical Western progression ... seems to rule out the possibility that there is an ideal or normal NDE scenario, except within a particular cultural context" (2001, p. 169). He nevertheless conceded vague crosscultural commonalities "in which individuals commonly use culturally-derived patterns to confabulate individualized death-process phenomena that serve common psychological functions" (2001, p. 177). Such commonalities may reflect common beliefs across societies, such as the idea of postmortem judgment for Earthly behavior, tied to an expectation or sense of being dead.

Journalists Hiroshi Tanami and Takashi Tachibana originally presented an investigation of 46 Japanese NDEs on NHK television (Morse, 1994, p. 70; Tachibana, 2000, Vol. 2, p. 90). Tachibana subsequently expanded the investigation to that of 243 NDEs (some individuals reported multiple NDEs) discussed in the Japaneselanguage book Rinshi taiken [Near death experience], which includes a section on the cultural differences between Japanese and other NDEs (Tachibana, 2000, Vol. 2, pp. 80-91). The study confirmed that Japanese NDErs often reported "seeing long, dark rivers and beautiful flowers, two common symbols that frequently appear as images in Japanese art" (Mauro, 1992, p. 57).

In a tabulated chart, 73 reports noted an affective component that was predominantly positive: 32 included being at peace and another 32 included feeling happy, while only 9 were fearful. Leaving the body was reported in $59 \mathrm{NDEs}$. Of 45 experiences of light, 16 were preceded by travel through a tunnel. Altogether 30 tunnel experiences were reported, implying that some experiences of light preceded (as in the Tatsuo Katayama case) or did not accompany tunnel experiences. Twenty-four NDEs included encountering darkness. In 153 accounts there was an encounter with others, most commonly with friends or relatives, but also occasionally with otherworldy beings or religious figures. Six NDEs included reviews of the events of the NDErs' lives. Landscape visions were most consistent between accounts, with reports of a field of flowers in 102 NDEs. Of the 82 NDEs featuring a border, 70 featured rivers, 6 featured a sea or lake, and 6 featured a gate or wall. In 72 accounts a reason for the NDErs' return was given: 
in 37 cases NDErs were "brought" back to life, in 23 they were told to return, and in 12 they chose to return (Tachibana, 2000, Vol. 2, p. 89).

Tachibana had noted that, unlike Western NDEs, the "light is not a personal (or personified) existence. ... [It] is an indescribably beautiful and natural light, yet is just light" (Tachibana, 2000, Vol. 2, p. 81, translated by $\mathrm{O}$. Carter), and that there was not a single instance of a Japanese NDEr having any sort of communication with the light. Nevertheless, occasional similarities between these Japanese NDEs and typical Western cases are fascinating exceptions to the general rule of cultural diversity. By the time of their collection in the $1990 \mathrm{~s}$, it is hard to say whether the popularization of common Western NDE features had influenced Japanese reports in this "East meets West" society. It is nevertheless notable that one Japanese NDEr recounted an experience dating back to World War II in which he began reviewing his childhood memories before he lost consciousness and had an NDE (Tachibana, 2000, Vol. 1, pp. 452-453).

In contrast to the Tachibana study, Yoshia Hata and collaborators at Kyorin University interviewed 17 patients who went into comas with "minimal signs of life" after heart attacks, strokes, asthma attacks, and drug poisoning. Eight of the 17 reported "dreams" where rivers and ponds were prominent, while the remaining 9 had no experiences. Five of the 8 NDErs reported "fear, pain and suffering," unlike typical NDEs from the West. While one of the patients from the Kyorin study reported repeatedly doing handstands in the shallows of a reservoir, a cardiac arrest survivor "saw a cloud filled with dead people. 'It was a dark, gloomy day. I was chanting sutras. I believed they could be saved if they chanted sutras, so that is what I was telling them to do"' (Hadfield, 1991, p. 11).

University of Zambia physician Nsama Mumbwe collected 15 NDE reports from Africa (Morse and Perry, 1992). In one case, after being attacked by a lioness protecting her cubs, a 60 -year-old truck driver reported that a highway opened up for him going endlessly into the sky, surrounded by stars; when he tried to go on to the highway, the stars blocked his way, and he stood there until the highway and stars disappeared. In another case, an 85-year-old stroke survivor reported: "I felt I was put into a big calabash [the hollow shell of a gourd] with a big opening. But somehow I couldn't get out of it. Then a voice from somewhere said to me, "be brave. Take my hand and come out. It is not yet your time to go"' (Morse and Perry, 1992, pp. 122-123). In the first case the man attributed his NDE to a "bad omen"; in the second the 
NDEr thought someone was trying to "bewitch her" into death. Clearly these were culture-bound interpretations of the experience; but what of the reports of what was experienced?

The single report of being caught inside a calabash with a large opening has been interpreted as the tunnel experience, but may indicate feeling caught inside something more like a bottle with only one opening. So we have a single African NDE that may or may not include a tunnel experience. There is little reason to describe the other African case where a "highway" through the stars opens up as either a tunnel experience or an OBE, since there is no indication of enclosure or of looking down on one's body.

In the South Pacific region known as Melanesia, anthropologist Dorothy Counts surveyed NDEs among the hunter-gatherer Kaliai, who described an NDE world "having factories and wage employment. ... that reminds me of the view approaching Los Angeles from the air" (Counts, 1983, p. 130). One Kaliai NDEr reported walking through a flower field to a road that forked in two. In each fork of the road a man was standing, trying to persuade the NDEr to come with him. The NDEr picked one of the forks at random and he and his guide traveled to a village where they climbed a ladder going up into a house floating in mid-air.

When they reached the top, a voice declared that the NDEr's time was yet to come and that a group of people would be summoned to take him back. The NDEr nevertheless caught a glimpse of what was inside the house, finding "some men working with steel, and some men building ships, and another group of men building cars" (Counts, 1983, p. 120). Once taken down the steps, the NDEr tried to return to the house, but found that with each attempt to approach its door, the house would turn as if on an axle so that the door moved elsewhere. When the NDEr could not find a road to follow to come back, a "beam of light" appeared that he walked along, and then he climbed down the steps, now finding "nothing but forest" at the bottom. The NDEr then reported: "So I walked along the beam of light, through the forest and along a narrow path. I came back to my house and re-entered my body and was alive again" (Counts, 1983, p. 120).

The NDE world of the Kaliai conformed to their cargo belief that white people were "spirit people" or ancestors who had returned from the dead. The Kaliai believed that spirits or ancestors gave technology to human beings, and that the afterlife was "rich with divinely given technology," including "factories, automobiles, highways, airplanes, 
European houses and buildings in great numbers, and manufactured goods" (Counts, 1983, p. 130). After discovering such major differences between Western and nonWestern descriptions, Counts concluded that the NDE world "often is a pleasant, happy place" whose content "varies and seems to be culturally defined," noting that Western NDErs often "see a beautiful garden, while Kaliai find an industrialized world of factories, highways, and urban sprawl" (Counts, 1983, p. 132). Counts also thought that this cultural variability indicated that OBEs and NDEs were probably just "the result of a psychological state. ... rather than an objectively experienced "life after death" (Counts, 1983, p. 132).

\section{How Consistent Are NDE Features Across Cultures?}

Despite a few core elements, descriptions of the world encountered during Western NDEs are nearly as variable as dreams. Even the identity of "the light" is variable: in some cases it has a personality, in others it does not; sometimes it has a specific identity that varies between people - a "bright angel" in one case, Jesus in another; in other cases it is a path, or simply the illumination of another world at the other end of a tunnel (Fox, 2003). Its function also varies significantly, "sometimes merely acting as a destination, sometimes lighting the way, sometimes judging, sometimes asking questions, and sometimes simply returning NDErs to where they came from" (Fox, 2003, p. 140).

Nevertheless, different near-death researchers have suggested that most of the following elements constitute core commonalities of the prototypical Western NDE: (1) feelings of peace or euphoria, lack of pain; (2) an out-of-body experience; (3) traveling through a tunnel or darkness from the physical world to an ostensibly transcendental realm; (4) encountering a sun-like light; (5) meeting others; (6) a life review where one's own life events are viewed or relived and sometimes judged; (7) seeing some sort of landscape; and (8) encountering a barrier or threshold between life and death.

Because most Western NDEs do not include all of these elements, I will define the prototypical Western NDE as an experience succeeding a real or perceived imminent physical threat containing at least two of the elements listed above. In nonWestern contexts an NDE should generally be understood as an ASC precipitated by either an expectation of dying or actual proximity to death. 
Consistency between different Western NDE accounts has been well established. But to determine whether their commonalities can be found in nonWestern NDEs, we must search existing nonWestern NDE accounts for elements of the prototypical Western NDE. Table 1 summarizes whether or not prototypical Western NDE elements were reported in 11 studies of nonWestern NDEs.

For the sake of fidelity, I have excluded from this crosscultural survey ambiguous NDE cases, unsoundly procured accounts, and case studies of just one account. For instance, whether various historical narratives are accounts of experiences by individuals who were dying or expected to die is unclear, or otherwise their status as unedited, first-person accounts rather than legendary inventions or accretions is dubious. I also excluded studies in which contemporary nonWestern accounts were derived solely from second-hand sources rather than from interviews with NDErs themselves. Finally, I have excluded data that are likely to have been contaminated by knowledge of Western NDE motifs, such as findings based on responses to advertisements in nonnative languages (for example, Blackmore, 1993b) or on composite accounts of Western NDEs presented to respondents beforehand (for example, Kellehear, Heaven, and Gao, 1990). It is nevertheless worth noting that none of the excluded nonWestern historical narratives (Bailey, 2001; Becker 1981, 1984; McClenon, 1991; Schorer, 1985; Wade 2003), second-hand accounts (Osis and Haraldsson, 1977), or single accounts (Berndt and Berndt, 1989; Gómez-Jeria, 1993; Kellehear, 2001; King, 1985) reported initial euphoria, transitional passage through a tunnel or darkness, encountering a sun-like light, or anything like the Western life review.

In 11 studies of seven nonWestern cultures (with three studies in India, two in Japan, and two in central Africa), very few elements of the prototypical Western NDE were universally present. Feelings of peace have only been reported in China and Japan, and unfortunately Feng Zhi-ying and Liu Jian-xun (1992) simply tabulated reported elements without including NDErs' narratives, making it impossible for us to determine how similar the Chinese elements were to prototypical Western ones. A single Thai NDEr did report "a deep sense of beauty" after an OBE, followed by walking down a road, encountering Yamatoots, and desperately trying to escape from them (www. altered-states.net).

OBEs were widely present, but absent from NDEs in Zambia, the Congo, and Melanesia, and only one OBE was reported in the 55 


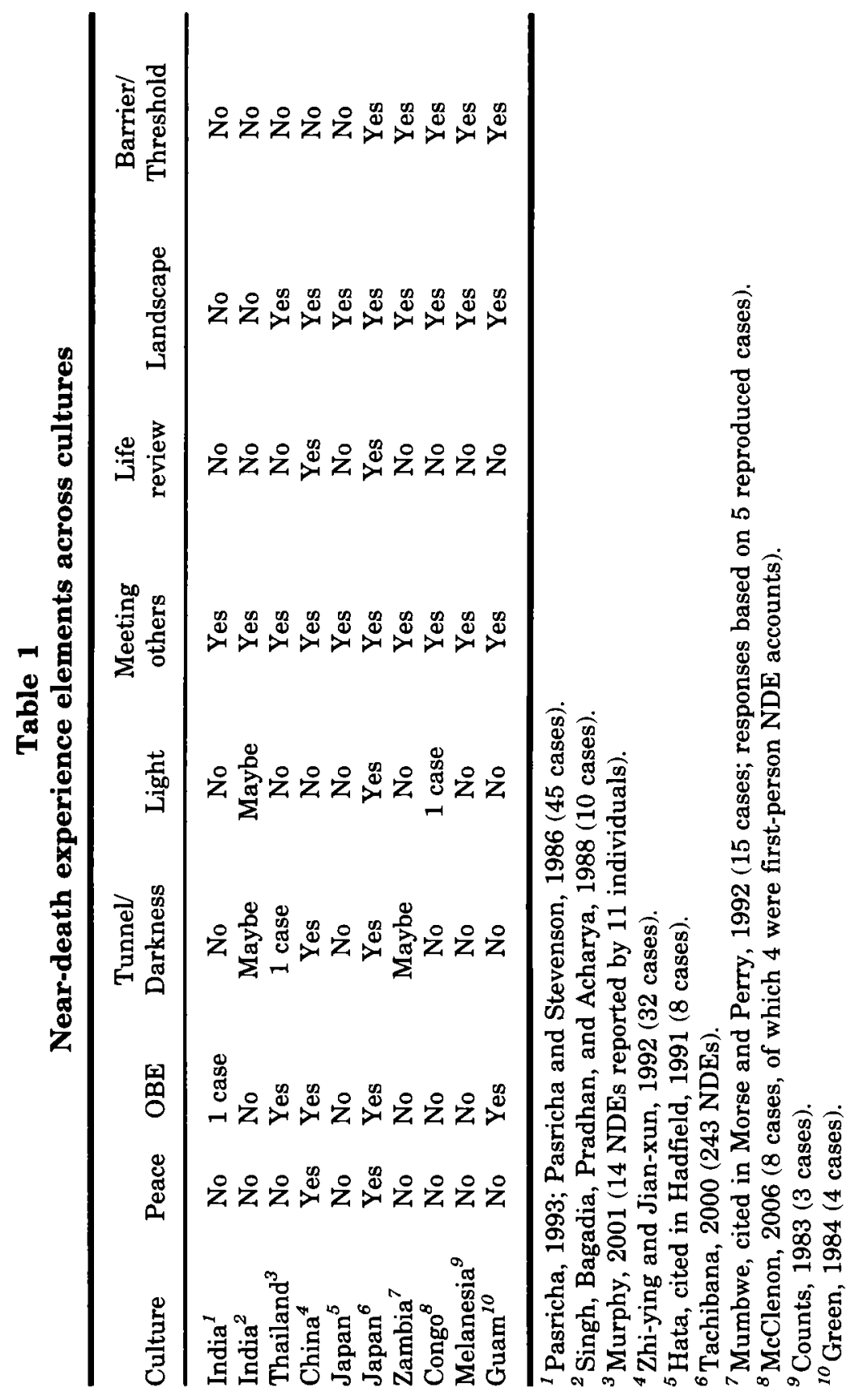


reports of NDEs from India. Moreover, the OBEs from Guam did not involve looking down on one's body or the area around it, but of out-ofbody "flights" to relatives living in America. Perhaps OBEs are not universal in NDEs because "not all cultures recognize OBEs as a herald of death" (Murphy, 2001, p. 171).

Tunnel experiences were limited virtually to China and Japan. One NDEr from Zambia may have reported one, depending on whether "calabash" should be interpreted as "tunnel," and in a tabulated chart three NDErs from India were paraphrased as reporting a "Feeling of intense darkness" that may or may not represent transitional passage (Singh, Bagadia, Pradhan, and Acharya, 1988 p. 303). In one Thai account an NDEr encountered a tunnel twice, first as a means to travel to Yama's hall of judgment, skipped due to "bad weather" (an elevator was taken instead), and then later to "access" the first level of Hell, where people with the "heads of chickens, buffalos, and cows" were encountered (www.altered-states.net); but Thai NDErs typically traversed roads in their NDEs (Murphy, 2001).

An experience of a sun-like light can be found among Japanese NDE reports, but otherwise only a Congo NDEr "saw a bright, dazzling light" (McClenon, 2006, p. 25), and an NDEr from India was paraphrased as reporting "Feeling of bright light and exploding firecrackers" (Singh, Bagadia, Pradhan, and Acharya, 1988, p. 303). A "light that was too bright to bear" was seen where "the cries of those being tortured" could be heard in the fourth level of Hell (reserved for arsonists) in a single Thai NDE (www.altered-states.net).

Meeting others was the only truly universal element found in every nonWestern NDE study, but it hardly lent support for the existence of any substantial core NDE across cultures, as it would not be surprising to find encounters with others in many hallucinatory human experiences. Moreover, it covered much variation, such as meeting deceased friends or relatives, strangers, religious figures, animals, living persons, and mythological creatures.

Viewing or reliving one's own life events was reported in NDEs only from China and Japan, although NDEs from India and Thailand often included readings of written records of the events of one's life, reflecting traditional Hindu and Thai beliefs (Murphy, 2001; Pasricha and Stevenson, 1986). Thai NDErs were often judged for specific actions and whether their good deeds outweighed their bad ones, but those affected by their actions (including a rooster and a turtle in one case) would testify for or against them. Outside of the limited Chinese 
and Japanese data, no "memory flashback" life reviews were reported by any nonWestern NDEr. One Melanesian NDEr reported witnessing a sorcerer being judged by loudspeaker and destroyed by fire during his NDE, but the NDEr himself never reviewed the events of his own life (Counts, 1983, pp. 121-122).

Encountering a landscape was nearly universal, but absent from NDEs in India, where NDErs typically entered an administrative office and were sent back because the clerks had been sent the wrong person. This motif was also found in Thai NDEs, occasionally preceding landscape visions. The most common features of the Thai NDE world were visions of Yama's palace, various tortures being inflicted on wrongdoers, and gardens or land strewn with jewels. But NDE landscapes varied considerably across cultures: bodies of water were prominent in NDEs from Japan; fields in NDEs from Guam; and brightly lit cities with factories, cars, roads, planes, and buildings in NDEs from Melanesia.

A barrier or threshold was reported in NDEs from Japan (a river, sea, gate, or wall), Zambia (stars blocking a highway), the Congo (a river), Melanesia (a house would turn if a door was approached), and Guam (a field entrance, a door, a hill in the distance to which the NDEr was prevented from traveling). In Thai NDEs, Yamatoots often forced NDErs back into their bodies after they were recognized to be the wrong person, but no barrier prevented them from going further, and no choice to stay or return was ever offered.

Of the eight prototypical Western NDE elements, only "meeting others" was truly universal in nonWestern cultures. Landscapes were nearly universal, but quite variable in their details. Even the OBE did not appear to be a universal NDE element, though it was more common than many of the other elements sought in nonWestern NDEs. Encountering a barrier one cannot cross was equally prevalent. The absence of feelings of peace, a clear tunnel experience, an experience of light, and a life review in almost all of the nonWestern NDE reports was surprising given their prominence in the prototypical Western NDE.

Presumably crosscultural consistency could be explained in terms of either similar neurological events or different encounters with the same afterlife reality. But crosscultural NDE studies demonstrate that many near-death researchers have simply assumed that the consistency between Western accounts was merely a specific instance of a crosscultural consistency. For instance, Paul Badham asserted 
that "What is seen [in NDEs] appears to be cross-cultural, but how it is named depends on the religious or non-religious background of the believer" (1997, p. 14). Though no prototypical Western NDEs were evident among his Western and nonWestern medieval afterlife narratives, James McClenon argued that what is at "issue between 'believers' and 'skeptics' is not whether common elements exist crossculturally," but whether that alleged fact "supports belief in life after death ... [or whether] commonalities within NDEs are produced by physiological factors associated with death trauma" (1991, p. 322). But existing crosscultural studies suggest that any crosscultural core consists of a very small number of elements. Thus sociologist Allan Kellehear suggested a very general and rather meager "core NDE" after his survey of nonWestern NDE accounts:

In every case discussed, deceased or supernatural beings are encountered. These are often met in another realm ... [which] is a social world not dissimilar to the one the percipient is from. The major difference is that this world is often much more pleasant socially and physically. Clearly, the consistency of these reports suggests that at least these two features of the NDE are indeed cross-cultural. (Kellehear, 1996, p. 33)

Of course, such variability does not undermine the survival hypothesis itself. It is entirely possible that an afterlife exists but that NDEs are not glimpses of it, a view similar to the Buddhist belief that the dying pass through several illusory bardo states generated by their own minds before entering the "real" afterlife (Fox, 2003). But it certainly undermines a survivalist interpretation of NDEs.

That "the major cross-cultural features of the NDE appear to include encountering other beings and other realms on the brink of death" (Kellehear, 1996, p. 34) is a surprisingly modest conclusion. Such little crosscultural consistency hardly cries out for either a neuroscientific or survivalist explanation. We would expect a real or perceived near-death crisis to produce a sensation of dying congenial to common hallucinations of deceased friends and relatives, religious figures prominent in one's own culture, and afterlife vistas that, aside from being a better place to live, appear exactly like the world of the living.

Fox suggested that a more specific core NDE could be discerned:

[I]t seems clear that certain motifs do recur within NDErs' testimonies with some regularity, both cross-culturally and preMoody. The presence of some kind of light motif seems very 
widespread ... as does the experience of a period of darkness en route or prior to it. (2003, p. 136)

While these do seem to be recurrent motifs across Western NDE accounts, there is little evidence that prototypical Western NDE motifs appear in nonWestern NDEs. NDEs from India, for example, are well documented yet markedly different from those reported in the West.

Traversing a tunnel or void toward a light is absent from most known nonWestern NDE accounts. In fact, it is notable that Kellehear did not include categories for "darkness" and "light" in his "Summary of Non-Western NDE Features" table (Kellehear, 1996).

Since far more differences than similarities have been found between Western and nonWestern accounts, the commonalities between different Western NDEs require a special explanation. What could possibly explain consistency between Western accounts but not crosscultural consistency?

Here a sociological explanation is called for rather than a neuroscientific or survivalist one. The most obvious suggestion is that the widespread dissemination of Raymond Moody's depiction of NDEs in his 1975 Life After Life produced consistency across Western NDE accounts. Selection bias (omitting incongruent reports) and interviewer bias (asking leading questions) might then account for the consistency between Moody's reports (Fox, 2003).

But Fox has revealed pre-Moody NDEs from the RERC archives, which contain more than 6,000 reports of religious experiences from 1925 to present (Fox, 2003). For instance, RERC account 2733 was written in August 1971 and referred to traveling down a long, whirling, black tunnel toward a bright light at the end, where an NDEr found herself floating blissfully in a warm, golden mist and saw faces coming out of the mist, smiling, and then fading away before "waking up" being slapped on both sides of the face (Fox, 2003, p. 118).

The evidence for consistency between Western NDEs is not limited to this single pre-Moody case. An NDE reported by J. W. Haddock in 1851 featured an OBE and life review (Crookall, 1964, p. 86). An 1889 NDE reported by Frederic Myers included an OBE and encountering a dark cloud, a dark pathway, other beings, and a border (Kelly, Greyson, and Kelly, 2006, p. 371). In 1935 G. B. Kirkland reported his own NDE, which included an OBE, life review, traveling through "a long tunnel" with a "tiny speck of light at the far end," seeing others traveling through the tunnel, encountering an uncrossable barrier, 
then finally returning to his body (Crookall, 1964, p. 89). And in 1970 an ineffable NDE marked by clarity of thought included an OBE, "travelling at great speed" sitting on something, noticing a "perfectly rectangular" but porous "pure white cloud-like substance" on an intercept course, and quite pleasantly "floating in a bright, pale yellow light" before ultimately regaining consciousness (MacMillan and Brown, 1971, pp. 889-890).

Moreover, at about the same time that Life After Life (Moody, 1975) was published, German minister Johann Christoph Hampe had been independently collecting NDE accounts from psychical research literature and the testimonies of mountain-climbing fall survivors (Fox, 2003). His German work was translated into English as To Die is Gain in 1979. Unlike Moody's accounts, Hampe's collection included "return trips" back through tunnels, did not include ringing noises, and rarely included encounters with deceased relatives. Nevertheless, Hampe independently found many prototypical Western NDE features: the realism of the experience, lucid thought during it, OBEs, tunnel experiences, experiences of light, life reviews, and transformations (Fox, 2003).

Finally, a study of the influence of Moody's model on American and British NDE accounts compared 24 reports of NDEs collected before 1975 with 24 demographic- and situation-matched post-1975 NDE accounts (Athappilly, Greyson, and Stevenson, 2006). If familiarity with the depiction of NDEs found in Life After Life generated widespread consistency between Western NDE accounts, one would expect post1975 accounts to "include higher frequencies of features included in Moody's synopsis than do [pre-1975] accounts" (Athappilly, Greyson, and Stevenson, 2006, p. 219). However, a systematic comparison found no statistically significant difference in the frequency of reports of 14 of Moody's 15 characteristic NDE features, with only "reports of a tunnel ... significantly more common in NDEs reported after 1975 than before" (Athappilly, Greyson, and Stevenson, 2006, p. 220).

The discovery of major elements of the prototypical Western NDE in pre-Moody NDE accounts thus rules out Moody's depiction of NDEs or that of subsequent researchers as the basis of the consistency between different Western NDE accounts. Nevertheless, we should be clear about the differences even as we acknowledge the commonalities, as Kellehear wisely cautioned:

[T] he full image of the NDE is an artifact of the composite picture put together by Moody and repeated endlessly in the NDE literature. Few 
people actually experience all of these images. ... Our understanding of the NDE has been shaped not by the diversity of NDEs but by a collective portrayal of features seldom occurring all together but immortalized by Moody's medical vignette. (Kellehear, 1996, p. 162)

Kellehear pointed out that about 66 percent of widows and 75 percent of parents who lose children experience bereavement hallucinations where their lost loved ones are briefly seen or heard. By contrast, in a recent prospective study only about one third (32 percent) of those who had NDEs reported meeting deceased persons, and only about a quarter ( 24 percent) reported OBEs. In fact, only two of the 10 tabulated elements were found in half or more of the NDErs: positive emotions ( 56 percent) and an awareness of being dead ( 50 percent) (van Lommel, van Wees, Meyers, and Elfferich, 2001). Thus even the sort of imagery we would expect to find in hallucinations near death is not nearly as common as our image of the prototypical Western NDE suggests.

Moreover, discrepancies between different researchers' accounts of the order of NDE elements - whether an OBE is directly followed by a tunnel experience or instead by otherworldly scenes, for instance - is an artifact of trying to "create a pattern out of the welter of various tunnels and paths, lights and presences, gardens and edifices" (Zaleski, 1987, p. 123). In other words, researchers have extracted features from several different NDE accounts from the West and put them back together into one coherent (but artificial) story where the stages of the NDE are clearly defined and follow a "developmental sequence" (Zaleski, 1987, p. 123). Nevertheless, there is little doubt that Western NDEs really are "patterned phenomena" marked by similar elements even if "all [of] these stages are not perceived by everyone and ... are not necessarily consecutive levels of experience" (Lindley, Bryan, and Conley, 1981, p. 105). Moreover, clichéd commonalities between dreams - dreams of falling or flying, for instance - do not seem nearly as similar between individuals as NDEs do.

Perhaps Western NDE motifs are found in some part of the Western cultural background other than the NDE literature since Moody. But then one is nagged by a poignant issue raised by Fox:

[I]n the cases where NDEs with classic features such as tunnels and lights are reported, we might wish to question where NDErs actually derive their cultural-linguistic NDE pattern from. ... For it is clear that such experiences, complete with recurring motifs such as 
traversing a period of darkness towards a light, do not represent part of any of the religious traditions of the West. (Fox, 2003, p. 117)

Specific NDE motifs are absent from standard depictions of the afterlife provided by Western religious traditions. But Irwin carried out a systematic survey of Western stereotypes of the afterlife to test the hypothesis that during "sudden confrontation with death people might draw upon their common cultural heritage to generate comparatively uniform hallucinatory images about a state of existence that is independent of the physical body" (Irwin, 1987, p. 1).

Irwin first considered the biblical depiction of Heaven offered in Revelation 21 , but noted that it was actually at variance with Western NDE motifs:

The difficulty here is that the biblical account is somewhat at odds with the descriptions of the afterlife realm given by subjects of the NDE. ... [T] The general public would be well aware of [the biblical] representation of heaven as a city of buildings and streets of pure gold and a surrounding high wall with [pearly] gates. In the NDE on the other hand, the post-mortem realm commonly is reported to comprise a pastoral setting, one with rolling green hills, trees, flowers, perhaps a stream and a blue sky above. (Irwin, 1987, pp. 1-2)

On the face of it "this disparity does not sit well with the view that the near-death experient's image of the afterlife springs largely from social conditioning" (Irwin, 1987, p. 2). However, biblical imagery did feature in some NDE reports, but more importantly, it is questionable "that the portrayal of heaven in Revelation 21 forms the popular stereotypical image [of the afterlife] in our culture" (Irwin, 1987, p. 2).

Consequently, Irwin set out to determine the most common Western visions of the afterlife by administering a questionnaire survey to 96 introductory psychology students concerning the appearance, inhabitants, and means of travel of the afterlife, as well as its auditory features. The most common Western images of the afterlife included a cosmic existence simultaneously everywhere and nowhere in the universe (40 percent), a pastoral scene of "lush green hills, trees, flowers and streams" (30 percent), and a formless void of pure being (29 percent) (Irwin, 1987, pp. 2-3). A mere 7 percent of respondents selected the biblical image, and 9 percent expected large gardens to figure prominently in the afterlife.

Irwin drew three key conclusions. First, there were several different Western visions of the afterlife. Second, the biblical image of Heaven was not widely held, and thus sociological sources of NDE motifs "can 
not be denied on the grounds that the account of the afterlife in NDEs fails to correspond to the biblical representation" (Irwin, 1987, p. 3). Finally, the image of the afterlife as a pastoral scene - an image often represented in NDEs - was quite commonplace, even though respondents indicated that "the pastoral stereotype generally is not based on familiarity with NDEs" (Irwin, 1987, p. 3; italics added).

Like the image of looking down upon the Earth from the clouds in the afterlife, a pastoral scene appears to have an obscure but clearly Western cultural source independent of NDE reports themselves. And in turn this image - like that of a garden or the pearly gates - appears to have influenced the content of some Western NDE reports. As Irwin noted, religious indoctrination was one possible source for the pastoral image: "the Bible frequently appeals to pastoral metaphors ... [and] Sunday School classes often include exposure to pictures of Christ standing in a grassy, sunlit field" (Irwin, 1987, pp. 3-4). And the "cosmic" image of the afterlife, which Irwin suggested was "rooted in diverse mystical and non-Christian traditions," appeared to be represented in a "meaningless void" experience in which a 28-yearold woman reported encountering a small group of jeering circles "clicking" back and forth from black to white, and vice versa, which she later discovered were Taoist yin-yang symbols, a symbol she likely was subconsciously aware of but had consciously forgotten about (Greyson and Bush, 1992, p. 102).

One prototypical Western NDE element may be represented by two items in Irwin's questionnaire (items 6 and 7), which combined indicated that a full 57 percent of respondents anticipated some sort of illuminating light in the afterlife (Irwin, 1987). If we combine being "bathed in perpetual sunshine" with being "illuminated by a soft, diffuse light with no apparent source," respondents anticipated illuminating light more than any other particular item concerning the appearance of the afterlife, and this was the only feature anticipated by a majority of the respondents. Though "illuminating light" may be too vague to be identified with it, an experience of light is a major Western NDE motif - perhaps the most prominent feature of NDEs in the popular imagination.

Though no other NDE elements were evident in Irwin's survey, OBEs appear to represent the most natural way to imagine what will happen to your soul immediately after the death of the body (Zaleski, 1996). Moreover, Heaven - which polls indicate is where the vast majority of people expect to end up after death (Gallup, 1992, p. 5) - is 
explicitly conceived of as a place of bliss and peace. Tunnels might be the most natural representation of transition for Westerners (Kellehear, 1996). And, as is evident in one of the creation accounts in Genesis, light is often associated with what is good in Judeo-Christian tradition, and God is conceived of as perfectly good. It is not much of a leap to associate God with light, and to think that God would be found on the other side of a transition between life and death. Individuals universally expect to meet others in the afterlife, and most contemporary religious traditions posit some sort of postmortem accounting or judgment of one's actions during Earthly life. Consequently, it is possible that NDErs are interpreting their experiences of specific physiological events in terms of their cultural expectations.

Interestingly, aside from Hieronymous Bosch's suggestive $14^{\text {th }}$ century painting "Ascent in the Empyrean" (which parallels Gustave Doré's 1868 painting "Vision of the Empyrean"), there is scant if any evidence of prototypical Western NDE motifs prior to the $19^{\text {th }}$ century. Classical and medieval "afterlife experience" motifs were markedly different from those found in contemporary NDEs (Bremmer, 2002; Zaleski, 1987). The legend of Er in Plato's Republic is often cited as the oldest recorded NDE, but like all of Plato's dialogues, it is entirely fictional, and in any case the only prototypical Western NDE motif possibly present was a vague OBE. In medieval accounts "most of the modern elements, such as the feelings of peace, the tunnel, the hovering above the body, the life review, and meetings with deceased relatives, or even brethren, are generally missing" (Bremmer, 2002, pp. 99-100).

Jan Bremmer surmised that contemporary NDE reports reflect a modern Western individualism absent from earlier afterlife accounts, and proposed this as a potential sociological source of prototypical Western NDE motifs. In addition to noting that the comparatively nonjudgmental nature of the contemporary life review contrasts sharply with medieval motifs, he asked: "Can it be that the relatively late $\left[19^{\text {th }}\right.$ century] appearance of reports of the life film have something to do with the development of the diorama and its reinforcement by the train in the nineteenth century, which enabled people to see a fast succession of scenes as they had never been able to do before?" (Bremmer, 2002, p. 102; bracketed comment added).

Kellehear suggested a possible cultural source for Western NDE imagery other than religious tradition:

The Velveteen Rabbit contains many of the classic images of the NDE. However, it is not unique in this respect. In The Wizard of $\mathrm{Oz}$, for 
example, Dorothy is transported inside the "tunnel" of a tornado to another place, where she meets the "good witch of the east." In Alice in Wonderland, Alice begins her adventure by a long fall down a dark rabbit hole. Children's literature is replete with tunnels, extraordinary beings, life reviews, flying experiences, and tales of reunion. (Kellehear, 1996, p. 153)

That Western NDE motifs might be derived from well-known children's stories would account for the considerable consistency between Western NDEs in the absence of evidence of significant crosscultural consistency. However, the similarities between the imagery described in children's stories and NDE imagery are superficial. Moreover, these stories do not concern what to expect when you die. Why would individuals who felt that they were dying derive NDE imagery from children's stories rather than better-known and more context-appropriate afterlife imagery from Western religious traditions?

Evidence of substantial consistency between different NDEs in the West but only trivial crosscultural consistency opens up two avenues for future research: a sociological search for more compelling links between NDE motifs and possible cultural sources within Western tradition; and more anthropological studies of NDEs in a greater variety of nonWestern cultures with larger sample sizes. The latter avenue is paramount: If more robust studies confirm the existence of little or no crosscultural consistency between NDE reports, the need to search for NDE motifs within Western tradition will become more pressing. But if more robust studies establish that specific NDE elements are crossculturally consistent, searching for a Western source of NDE motifs will become unnecessary, and knowledge of which NDE motifs are universal or at least widespread would provide us with better data to develop and test specific neuroscientific explanations of NDEs.

Given that at least some NDEs are known to be hallucinations (Augustine, 2007), should future crosscultural studies uncover universal and well-defined NDE elements, such precise and widespread commonalities would be best explained in neuroscientific terms. Alternatively, if extensive studies fail to uncover substantial crosscultural consistency between NDE accounts, a sociological explanation for solely Western commonalities would be required.

In any case, our best evidence shows that a survivalist explanation will not do. Despite the commonalities, there is considerable variation in the content of different NDEs. While one person actually sees Jesus, 
another sees the Hindu god Yamraj. The glossed explanation that different people simply describe or interpret the same experience in different ways - an encounter with the same "being of light," for instance - does not do justice to the actual NDE reports that have been collected, where Jesus and Yamraj have very different appearances and functions.

When NDErs provide very different descriptions of their experiences, there is no reason to assume that different NDErs are simply using different labels to describe the same experience. The most straightforward reason why different NDErs would describe their experiences in different ways is because they actually have very different experiences. The standard survivalist explanation - that despite appearances, different NDE reports really are similar - is simply an ad hoc stipulation.

This principle is widely assumed because different people would naturally be expected to report similar experiences if they were traveling to the same afterlife environment. Of course, the greater the diversity between different NDE accounts, the less credible the NDE consistency argument for survival, and thus the greater the pressure to portray different NDE accounts as more consistent than they first appear.

But NDE reports themselves do not support this principle, and often contradict it, even when considering supposedly core elements. Kellehear, for example, suggested that perhaps:

tunnel experiences are not cross-cultural but that a period of darkness may be. This darkness is then subject to culture-specific interpretations: a tunnel for Westerners, subterranean caverns for Melanesians, and so on. NDErs who do not report darkness may not view this aspect of the experience as an important part of their account or narrative. ... Why is the frequently reported sensation of traveling through a darkness by Western NDErs so often described as a tunnel experience? (Kellehear, 1996, pp. 35-36)

But the most straightforward interpretation of apparent diversity is actual diversity: that Westerners actually see tunnels in their experiences, while Melanesians see underground caverns. But since acknowledging actual diversity tends to undermine arguments for survival based on NDE commonalities, it is not surprising that researchers sympathetic to survival after death tend to interpret markedly different NDE reports in terms of the "same experience, different descriptions" principle. 
After finding several accounts of visiting outer space in the RERC archives, Fox asserted that many NDErs "were found to use the descriptor 'space' to describe the episode of darkness" (Fox, 2003, p. 265). Soon after he wrote: "The significance of the word 'space' to describe the darkness encountered in these experiences will be returned to" (Fox, 2003, p. 265). Finally, Fox skewed what was actually reported by NDErs by concluding that many of his RERC respondents "describe an encounter with some sort of darkness motif in terms seemingly suggestive of a visit to outer space" (Fox, 2003, p. 277).

Fox was speaking as if he knew ahead of time what these different people had experienced. But of course assuming that these reports were describing the same thing regardless of what they actually said begs the question, for that is the very issue of contention. Fox asserted that the episode of darkness was what was encountered, but that it was being described as "space" - but how did he know that it was not space that was actually encountered? Of course he did not know; he just assumed.

If we look at such NDErs' actual testimonies, one person reports encountering a tunnel while another reports encountering space. But should we not take each at his word instead of assuming that both are encountering the same darkness in order to fit our artificial NDE models? Just because space, tunnels, and underground caverns are dark does not mean that astronauts, motorists, and miners are using different terminology to describe traveling to the same place. One wonders just how much consistency is being derived from NDE reports rather than being read into them.

Worse still, many NDErs report encountering tunnels with physical properties, such a being made of bricks. Consider a case reported by Peter and Elizabeth Fenwick:

[T] he tunnel was made of polished metal, jointed and held together with something like rivets. ... I felt it should have been more ethereal somehow. ... My feet felt like lead but as I walked back to life the light threw my shadow on to the floor of the tunnel as I walked away from it. (Fenwick and Fenwick, 1997, p. 55)

Clearly a case like this could not simply be a description or interpretation of a mere "episode of darkness." More plausibly, different NDErs experience different things: traveling down tunnels, floating in "outer space," walking down a road, being caught in a calabash, and so on. 
Greyson has conceded stark crosscultural variation, but argued that "experiences which conflict with [NDErs'] specific religious and personal expectations of death" challenge the view "that NDEs are products of imagination, constructed from one's personal and cultural expectations" (Greyson, 2000, p. 332). However, his argument fails, and in any case a few cases of apparent "cultural dissonance" in NDEs certainly does not outweigh an abundance of evidence for the predominant role of expectation in NDEs from different cultures, such as those from India and Thailand.

Greyson (2000) cited the case of the Jewish NDEr Chaim Ralbag, who experienced an unusual cardiac arrest NDE in which he was falling into a dark void a high speed, hovering over a sea of nothingness, then pushing himself back out of the void. As he traveled upward through the void, his surroundings were illuminated, and eventually he saw his body lying on the ground (Abramovitch, 1988). He then had an experience of light, communicated with another being, and encountered his deceased father and brother, before finally hovering over his body and then waking up in the recovery room.

Henry Abramovitch offered two reasons why expectation could not account for the content of Ralbag's NDE. First, Ralbag was taught that the biblical Adam would appear to the dying, but this did not happen in his NDE. Second, he was taught that he would face judgment in the afterlife, but had no life review (Abramovitch, 1988). That an individual's visions of the afterlife do not exactly mirror what he was taught, however, hardly requires an encounter with the afterlife to explain it.

The real issue is whether such experiences conflict with an individual's personal expectations. But what a person consciously claims to expect of the afterlife says nothing of his subconscious expectations, and many individuals do not simply accept whatever dogma was taught to them in childhood, but grow to elaborate upon, modify, or reject what they were taught.

Murphy found clear evidence of this in an NDE reported by the Chinese-Thai Kodien. During his NDE,

He noticed that some groups [of deceased people] had food and water, while others had only piles of ash. ... His friend said that those who made merit by donating food to the monks during their lives would have food [in the afterlife], while those who only helped their own ancestors (by burning joss-paper replicas of food according to Chinese tradition) would only have piles of ash. Kodien realized that his friend was suggesting that he create merit according to the Thai custom, but 
during his life he had not believed in the practice. He had thought that the Chinese forms of religious observance were better, and had only made joss-paper offerings. (Murphy, 2001, pp. 168-69)

Here an NDE was shaped almost entirely by cultural influences, yet the NDEr experienced something contrary to his conscious beliefs. But this "cultural dissonance" did not imply that this NDE was not expectation-driven, as Murphy explained:

His NDE seemed to reveal that he was actually more drawn to the Thai ways of creating merit than to those of his own family and subculture. ... [This] suggests that it is not culture alone that determines NDE phenomenology. Rather, NDEs may be determined more by one's expectations concerning what death will be like, even when those expectations are held subconsciously or are influenced by more than one culture. (Murphy, 2001, p. 169)

Greyson went on to argue that "children too young to have received substantial cultural and religious conditioning about death report the same kinds of [NDEs] as do adults" (Greyson, 2000, p. 332). But how young is "too young" for a child to be affected by cultural conditioning? Children younger than 3 years old clearly learn concepts from sources as ubiquitous as television advertising. Moreover, how confident can we be that children's NDE reports have not been contaminated by parental influence or interviewer feedback? Children are particularly susceptible to influence from authority figures and typically have much richer imaginative lives than adults. The risk of direct contamination from parents or researchers could be minimized by closing the gap between the time of the NDE itself and when it is reported, but such information is rarely published.

\section{Conclusion}

Taken as a whole, various psychophysiological correlates of OBEs and NDEs make little sense if consciousness is literally projected outside of the body during such experiences. Though more definitive research is needed, data suggesting that one's psychological state or medical condition affects the content of one's NDE undermine the notion that NDEs are instances of literal mind-body separation.

On the hypothesis that something leaves the body during OBEs and NDEs, for instance, one would not naturally anticipate that fear alone would be able to prompt a double to detach from or return to the body, 
though one could certainly amend that hypothesis to incorporate that finding after the fact. By contrast, extreme fear might very well be expected to prompt an individual to dissociate in order to "escape" from real or perceived impending trauma. Similarly, medical factors affecting a person's brain state would not be expected to influence the course of a "brain-free" experience, and yet we find medical influences on the content of OBEs and NDEs. Finally, there are several links between NDEs and temporal lobe signs, where such signs are not taken to be indicative of mind-body separation in other contexts. Given its greater predictive power, a psychophysiological explanation is clearly a better explanation of such facts than a survivalist or dualistic one.

Additionally, though different Western NDEs contain several recurrent and well-defined commonalities, very few and only broadly defined commonalities have been found crossculturally. This is problematic for a survivalist interpretation because a substantial crosscultural core would be expected if different NDErs were literally traveling, by the same means (leaving their bodies) and through the same route (passage through a tunnel or darkness toward a light), to transcendental destinations. Instead, we find a variety of culturespecific NDE templates with only locally well-defined commonalities. This severely undercuts the survivalist argument that NDE commonalities result from different NDErs undergoing the same journey; and in virtue of their common humanity, NDErs would not be expected to take different journeys to different places after death merely because of where and when they lived while on Earth.

Due to this implication, near-death researchers sympathetic to survival have typically assumed that cultural variation in the phenomenology of NDEs is only superficial. But the crosscultural data that have been collected do not bear this out. In fact, what is superficial is the strained explanation that, despite appearances, NDErs from different cultures encounter the same sorts of things but simply describe them differently. A quick glance at the content of nonWestern NDE reports demonstrates that this explanation is patently false: the NDE elements from different cultures have starkly different visual appearances and narrative roles, even in outline.

The available evidence implies that NDE motifs found in different cultures, including those found in the West, have sociological sources. Alternatively, though, this pattern could simply reflect the paucity of the available nonWestern NDE data. If so, the prototypical Western 
NDE would actually represent a substantial crosscultural core of NDE elements reflecting, perhaps, common neurophysiological processes. Only future research can definitively resolve this issue.

\section{References}

Abramovitch, H. (1988). An Israeli account of a near-death experience: A case study of cultural dissonance. Journal of Near-Death Studies, 6, 175-184.

Alvarado, C. S. (2000). Out-of-body experiences. In E. Cardeña, S. J. Lynn, and S. Krippner (eds.), The varieties of anomalous experience: Examining the scientific evidence (pp. 183-218). Washington, DC: American Psychological Association.

Alvarado, C. S., and Zingrone, N. L. (1994). Individual differences in aura vision: Relationships to visual imagery and imaginative-fantasy experiences. European Journal of Parapsychology, 10, 1-30.

Athappilly, G., Greyson, B., and Stevenson, I. (2006). Do prevailing societal models influence reports of near-death experiences? A comparison of accounts reported before and after 1975. Journal of Nervous and Mental Disease, 194, 218-222.

Augustine, K. (2007). Near-death experiences with hallucinatory features. Journal of Near-Death Studies, 26, 3-31.

Badham, P. (1997). Religious and near-death experience in relation to belief in a future life. Mortality, 2, 7-21.

Bailey, L. W. (2001). A "little death": The near-death experience and Tibetan delogs. Journal of Near-Death Studies, 19, 139-159.

Becker, C. B. (1981). The centrality of near-death experiences in Chinese Pure Land Buddhism. Anabiosis: The Journal of Near-Death Studies, 1, 154-170.

Becker, C. B. (1984). The pure land revisited: Sino-Japanese meditations and near-death experiences of the next world. Anabiosis: The Journal of Near-Death Studies, 4, 51-68.

Berndt, R. M., and Berndt, C. H. (1989). The speaking land: Myth and story in aboriginal Australia. Harmondsworth, England: Penguin.

Blackmore, S. (1984). A postal survey of OBEs and other experiences. Journal of the Society for Psychical Research, 52, 225-244.

Blackmore, S. (1987). Where am I?: Perspectives in imagery and the out-of-body experience. Journal of Mental Imagery, 11, 53-66.

Blackmore, S. (1993a). Dying to live: Near-death experiences. Buffalo, NY: Prometheus.

Blackmore, S. (1993b). Near-death experiences in India: They have tunnels too. Journal of Near-Death Studies, 11, 205-217.

Bremmer, J. N. (2002). The rise and fall of the afterlife. New York, NY: Routledge.

Britton, W. B., and Bootzin, R. R. (2004). Near-death experiences and the temporal lobe. Psychological Science, 15, 254-258.

Cook, A. M., and Irwin, H. J. (1983). Visuospatial skills and the out-of-body experience. Journal of Parapsychology, 47, 23-35.

Council, J. R., Greyson, B., and Huff, K. D. (1986). Fantasy-proneness, hypnotizability, and reports of paranormal experiences. Paper presented at the annual convention of the American Psychological Association, San Francisco, CA,

Counts, D. A. (1983). Near-death and out-of-body experiences in a Melanesian society. Anabiosis: The Journal of Near-Death Studies, 3, 115-135.

Crookall, R. (1964). More astral projections: Analyses of case histories. London, England: Aquarian Press.

Devinsky, O., Feldmann, E., Burrowes, K., and Bromfield, E. (1989). Autoscopic phenomena with seizures. Archives of Neurology, 46, 1080-1088. 
Drab, K J. (1981). The tunnel experience: Reality or hallucination? Anabiosis: The Journal of Near-Death Studies, 1, 126-152.

Fenwick, P., and Fenwick, E. (1997). The truth in the light: An investigation of over 300 near-death experiences. New York, NY: Berkley.

Fox, M. (2003). Religion, spirituality and the near-death experience. New York, NY: Routledge.

Fox, O. (1962). Astral projection: A record of out-of-body experiences. New Hyde Park, NY: University Books.

French, C. C. (2005). Near-death experiences in cardiac arrest survivors. In S. Laureys (ed.), The boundaries of consciousness: Neurobiology and neuropathology (pp. 351367). New York, NY: Elsevier Science.

Gallup, G. (1992). The Gallup poll: Public opinion 1991. Wilmington, DE: Scholarly Resources.

Glicksohn, J. (1990). Belief in the paranormal and subjective paranormal experience. Personality and Individual Differences, 11, 675-683.

Gómez-Jeria, J. S. (1993). A near-death experience among the Mapuche people. Journal of Near-Death Studies, 11, 219-222.

Green, J. T. (1984). Near-death experiences in a Chammorro culture. Vital Signs, 4(1-2), 6-7.

Greyson, B. (2000). Near-death experiences. In E. Cardeña, S. J. Lynn, and S. Krippner (eds.), The varieties of anomalous experience: Examining the scientific evidence (pp. 315-352). Washington, DC: American Psychological Association.

Greyson, B., and Bush, N. E. (1992). Distressing near-death experiences. Psychiatry, 55, 95-110.

Greyson, B., and Stevenson, I. (1980). The phenomenology of near-death experiences. American Journal of Psychiatry, 137, 1193-1196.

Hadfield, P. (1991). Japanese find death a depressing experience. New Scientist, 132, 11.

Hampe, J. C. (1979). To die is gain: The experience of one's own death. London, England: Darton, Longman and Todd.

Holden, J. M., Long, J., and MacLurg, J. (2006). Out-of-body experiences: All in the brain? Journal of Near-Death Studies, 25, 99-107.

Hunt, H. T., Gervais, A., Shearing-Johns, S., and Travis, F. (1992). Transpersonal experiences in childhood: An exploratory empirical study of selected adult groups. Perceptual and Motor Skills, 75, 1135-1153.

Irwin, H. J. (1985a). Flight of mind: A psychological study of the out-of-body experience. Metuchen, NJ: Scarecrow Press.

Irwin, H. J. (1985b). Parapsychological phenomena and the absorption domain. Journal of the American Society for Psychical Research, 79, 1-11.

Irwin, H. J. (1986). Perceptual perspective of visual imagery in OBEs, dreams, and reminiscence. Journal of the Society for Psychical Research, 53, 210-217.

Irwin, H. J. (1987). Images of heaven. Parapsychology Review, 18, $1-4$.

Irwin, H. J. (1993). The near-death experience as a dissociative phenomenon: An empirical assessment. Journal of Near-Death Studies, 12, 95-103.

Irwin, H. J. (1999). An introduction to parapsychology $\left(3^{\text {rd }}\right.$ ed). Jefferson, NC: McFarland.

Irwin, H. J. (2000). The disembodied self: An empirical study of dissociation and the outof-body experience. Journal of Parapsychology, 64, 261-277.

Kellehear, A. (1996). Experiences near death: Beyond medicine and religion. New York, NY: Oxford University Press.

Kellehear, A. (2001). An Hawaiian near-death experience. Journal of Near-Death Studies, 20, 31-35.

Kellehear, A., Heaven, P., and Gao, J. (1990). Community attitudes toward near-death experiences: A Chinese study. Journal of Near-Death Studies, 8, 163-173. 
Kelly, E. W., Greyson, B., and Kelly, E. F. (2006). Unusual experiences near death and related phenomena. In Kelly, E. F., Kelly, E. W., Crabtree, A., Gauld, A., Grosso, M., and Greyson, B., Irreducible mind: Toward a psychology for the 21st century (pp. 367-421). Lanham, MD: Rowman and Littlefield.

King, M. (1985). Being pakeha: An encounter with New Zealand and the Maori renaissance. Auckland, New Zealand: Hodder and Stoughton.

Kohr, R. (1983). Near-death experiences, altered states, and psi sensitivity. Anabiosis: The Journal of Near-Death Studies, 3, 157-176.

Lindley, J. H., Bryan, S., and Conley, B. (1981). Near-death experiences in a Pacific Northwest American population: The Evergreen Study. Anabiosis: The Journal of Near-Death Studies, 1, 104-124.

MacMillan, R. L., and Brown, K. W. G. (1971). Cardiac arrest remembered [Letter]. Canadian Medical Association Journal, 104, 889-890.

Makarec, K., and Persinger, M. A. (1985). Temporal lobe signs: Electroencephalographic validity and enhanced scores in special populations. Perceptual and Motor Skills, 60, 831-842.

Mauro, J. (1992, July/August). Bright lights, big mystery. Psychology Today, 54-57, 80-82.

McClenon, J. (1991). Near-death folklore in medieval China and Japan: A comparative analysis. Asian Folklore Studies, 50, 319-342.

McClenon, J. (2006). Kongo near-death experiences: Cross-cultural patterns. Journal of Near-Death Studies, 25, 21-34.

McCreery, C., and Claridge, G. (1995). Out-of-the-body-experiences and personality. Journal of the Society for Psychical Research, 60, 129-148.

McCreery, C., and Claridge, G. (1996). A study of hallucination in normal subjects: I. Self-report data. Personality and Individual Differences, 21, 739-747.

Moody, R. A. (1975). Life after life: The investigation of a phenomenon-survival of bodily death. Covington, GA: Mockingbird Books.

Morse, M. (1994). Near death experiences and death-related visions in children: Implications for the clinician. Current Problems in Pediatrics, 24, 55-83.

Morse, M., and Perry, P. (1992). Transformed by the light: The powerful effect of neardeath experiences on people's lives. New York, NY: Villard.

Murphy, T. (2001). Near-death experiences in Thailand. Journal of Near-Death Studies, $19,161-178$.

Murray, C., and Fox, J. (2005). The out-of-body experience and body image: Difference between experients and nonexperients. Journal of Nervous and Mental Disease, 193, 70-72.

Myers, S. A., Austrin, H. R., Grisso, J. T., and Nickeson, R. C. (1983). Personality characteristics as related to the out-of-body experience. Journal of Parapsychology, 47, 131-144.

Neppe, V. M. (2002). "Out-of-body experiences" (OBEs) and brain localisation. A perspective. Australian Journal of Parapsychology, 2, 85-96.

Noyes, R., and Kletti, R. (1976). Depersonalization in the face of life threatening danger: A description. Psychiatry, 39, 19-27.

Osis, K., and Haraldsson, E. (1977). At the hour of death: The results of research on over 1000 afterlife experiences. New York, NY: Avon.

Owens, J. E., Cook, E. W., and Stevenson, I. (1990). Features of "near-death experience" in relation to whether or not patients were near death. Lancet, 336, 1175-1177.

Pasricha, S. (1993). A systematic survey of near-death experiences in south India. Journal of Scientific Exploration, 7, 161-171.

Pasricha, S., and Stevenson, I. (1986). Near-death experiences in India: A preliminary report. Journal of Nervous and Mental Disease, 174, 165-174.

Richards, P., and Persinger, M. A. (1991). Temporal lobe signs, the Dissociative Experiences Scale and the Hemispheric Quotient. Perceptual and Motor Skills, 72, 1139-1142. 
Ring, K., and Rosing, C. J. (1990). The Omega Project: An empirical study of the NDEprone personality. Journal of Near-Death Studies, 8, 211-239.

Schorer, C. E. (1985). Two Native North American near-death experiences. Omega: Journal of Death and Dying, 16, 111-113.

Singh, A. R., Bagadia, V. N., Pradhan, P. V., and Acharya, V. N. (1988). Death, dying and near death experience. Indian Journal of Psychiatry, 30, 299-306.

Stevenson, I., and Cook, E. W. (1995). Involuntary memories during severe physical illness or injury. Journal of Nervous and Mental Disease, 183, 452-458.

Tachibana, T. (2000). Rinshi taiken [Near death experience] (2 vols.). Tokyo, Japan: Bungei Shunjusha.

Tong, F. (2003). Out-of-body experiences: From Penfield to present. Trends in Cognitive Science, 7, 104-106.

Twemlow, S. W., and Gabbard, G. O. (1984). The influence of demographic/psychological factors and preexisting conditions on the near-death experience. Omega, 15, 223-235.

Twemlow, S. W., Gabbard, G. O., and Coyne, L. (1982). A multivariate method for the classification of preexisting near-death conditions. Anabiosis: The Journal of NearDeath Studies, 2, 132-139.

van Lommel, P., van Wees, R., Meyers, V., and Elfferich, I. (2001). Near-death experiences in survivors of cardiac arrest: A prospective study in the Netherlands. Lancet, 358, 2039-2045.

Vuilleumier, P., Despland, P. A., Assal, G., and Regli, F. (1997). Voyages astraux et hors du corps: héautoscopie, extase et hallucinations expérientielles d'origine épileptique [Out-of-body and astral journeys: Heautoscopy, ecstasis and experimental hallucinations of epileptic origin]. Revue Neurologique, 153, 115-119.

Wade, J. (2003). In a sacred manner we died: Native American near-death experiences. Journal of Near-Death Studies, 22, 83-116.

Wilson, S. C., and Barber, T. X. (1983). The fantasy-prone personality: Implications for understanding imagery, hypnosis, and parapsychological phenomena. In A. A. Sheikh (ed.), Imagery: Current theory, research, and application (pp. 340-387). New York, NY: Wiley.

Zaleski, C. (1987). Otherworld journeys: Accounts of near-death experience in medieval and modern times. New York, NY: Oxford University Press.

Zaleski, C. (1996). The life of the world to come: Near-death experience and Christian hope. New York, NY: Oxford University Press.

Zhi-ying, F., and Jian-xun, L. (1992). Near-death experiences among survivors of the 1976 Tangshan earthquake. Journal of Near-Death Studies, 11, 39-48. 